\title{
Impaired energetics and normal myocardial lipids in rheumatoid arthritis and systemic lupus erythematosus: a phosphorous and proton magnetic resonance spectroscopy and cardiovascular magnetic resonance study
}

\author{
Ntobeko A Ntusi ${ }^{1,2^{*}}$, Cameron Holloway ${ }^{1,5}$, Jane M Francis ${ }^{1}$, Anne Davis ${ }^{1}$, Eylem Levelt', Stefan K Piechnik ${ }^{1}$, \\ Vanessa M Ferreira ${ }^{1}$, Paul M Matthews ${ }^{3}$, Paul B Wordsworth ${ }^{4}$, Theodoros D Karamitsos ${ }^{1}$, Stefan Neubauer ${ }^{1}$
}

From 18th Annual SCMR Scientific Sessions

Nice, France. 4-7 February 2015

\section{Background}

Rheumatoid arthritis (RA) and systemic lupus erythematosus (SLE) commonly involve the cardiovascular system and are associated with significant morbidity and mortality, driven by cardiovascular inflammation, microvascular and diastolic dysfunction and fibrosis. Cardiovascular magnetic resonance (CMR) can assess non-invasively cardiac function, strain, ischaemia, altered vascular function, perfusion, inflammation and fibrosis; magnetic resonance spectroscopy (MRS) provides further insights into the status of myocardial energetics and lipidosis. To date, there have been no cardiovascular MRS studies in RA and SLE patients. We hypothesised that RA and SLE would be associated with impaired myocardial energetics and lipidosis.

\section{Methods}

The study population consisted of 16 RA patients (10 female; mean age $51 \pm 13$ years), 13 SLE patients (13 female; mean age $43 \pm 9$ years) and 12 age- and sexmatched controls (8 female; mean age $49 \pm 15$ years). Patients with previously known cardiovascular disease (CVD) were excluded. Participants underwent CMR at $1.5 \mathrm{~T}$ and the assessments included cine, tagging, T1 mapping, T2-weighted, perfusion, late gadolinium $\left(0.15 \mathrm{mmol} / \mathrm{kg}\right.$ gadoteric acid - Dotarem $\left.{ }^{\circledR}\right)$ imaging and ECV quantification. Further assessments included

${ }^{1}$ OCMR, Division of Cardiovascular Medicine, University of Oxford, Oxford, UK Full list of author information is available at the end of the article proton $\left({ }^{1} \mathrm{H}\right)$ and phosphorous $\left({ }^{31} \mathrm{P}\right)$ MRS at $3 \mathrm{~T}$. Comorbid status, disease activity index and duration of disease were recorded for each subject.

\section{Results}

Patients were well-matched with controls for sex, age and comorbidity (Table 1). The DAS28-CRP was $5 \pm 1$ in RA and the SLEDAI was 7 (4-11), respectively, indicating ongoing disease activity. There were no differences in left ventricular (LV) size, mass and systolic function between RA and SLE patients when compared to controls (Table 2). Consistent with previously described findings, there were significant differences in strain, and tissue characteristics between the patients and controls. The PCr/ATP ratio was reduced in patients and measured $2.0 \pm 0.3,1.5$ \pm 0.3 and $1.4 \pm 0.3(\mathrm{p}=0.02)$ in controls, RA and SLE, respectively. No differences were found in cardiac lipid content when RA and SLE patients were compared to controls. PCr/ATP ratio correlated with presence of LGE ( $\mathrm{R}-0.38, \mathrm{p}=0.14)$, MPRI ( $\mathrm{R} 0.61, \mathrm{p}<0.001)$, LA size ( $\mathrm{R}$ $-0.32, \mathrm{p}=0.04)$, ECV $(\mathrm{R}-0.46, \mathrm{p}=0.006)$ and volume fraction of $\mathrm{T} 1>990 \mathrm{~ms}(\mathrm{R}-0.73, \mathrm{p}<0.001)$.

\section{Conclusions}

In RA and SLE patients (with no overt cardiovascular disease) myocardial energetics are impaired, likely due to abnormal mitochondrial dysfunction. Abnormal myocardial energetics are associated with indices of 
Table 1 Baseline characteristics

\begin{tabular}{|c|c|c|c|c|}
\hline & Controls $\mathrm{N}=12$ & $\mathrm{RA} N=16$ & SLE N=13 & $P$ value \\
\hline Female sex, n (\%) & $8(67)$ & $10(63)$ & $13(100)$ & 0.05 \\
\hline Age, years & $49 \pm 15$ & $51 \pm 13$ & $43 \pm 9$ & 0.18 \\
\hline Hypertension, n (\%) & 0 & $3(19)$ & $2(16)$ & - \\
\hline Diabetes, n (\%) & 0 & 0 & $1(8)$ & - \\
\hline Hyperlipidaemia, n (\%) & 0 & $3(19)$ & 0 & - \\
\hline $\mathrm{BMl}, \mathrm{kg} / \mathrm{m} 2$ & $24 \pm 3$ & $25 \pm 3$ & $27 \pm 5$ & 0.12 \\
\hline Chloroquine, n (\%) & $\mathrm{N} / \mathrm{A}$ & $11(69)$ & $11(85)$ & - \\
\hline Methotrexate, n (\%) & N/A & $15(94)$ & $1(8)$ & - \\
\hline Prednisolone, n (\%) & $\mathrm{N} / \mathrm{A}$ & $2(13)$ & $9(69)$ & - \\
\hline Leflunomide, n (\%) & N/A & $3(19)$ & $1(8)$ & - \\
\hline Sulfasalazine, n (\%) & $\mathrm{N} / \mathrm{A}$ & $3(19)$ & 0 & - \\
\hline Azathioprine, n (\%) & N/A & 0 & $4(31)$ & - \\
\hline Rituximab, n (\%) & N/A & $2(13)$ & $1(8)$ & - \\
\hline DAS28-CRP & N/A & $5 \pm 1$ & N/A & - \\
\hline SLEDAI (median, IQR) & N/A & N/A & $7(4-11)$ & - \\
\hline ESR, mm/hr (median, IQR) & N/A & $7(5-10)$ & $10(4-16)$ & - \\
\hline CRP, mg/L (median, IQR) & $1(1-1)$ & $5(2-7)$ & $4(2-6)$ & $<0.001$ \\
\hline Duration of disease, years (median, IQR) & $\mathrm{N} / \mathrm{A}$ & $11(7-14)$ & $13(6-16)$ & - \\
\hline Duration of DMARDs, years (median, IQR) & N/A & $7(5-11)$ & $8(3-11)$ & - \\
\hline
\end{tabular}

Continuous data are mean \pm SD unless otherwise indicated. Categorical data are frequency (percent) unless otherwise indicated. BMI, body mass index; CRP, Creactive protein; DAS28-CRP (rheumatoid arthritis disease activity index incorporating 28 swollen and tender joint count as well as the C-reactive protein); ESR, erythrocyte sedimentation rate; IQR, interquartile range; RA, rheumatoid arthritis; SLE, systemic lupus erythematosus; SLEDAl, systemic lupus erythematosus disease activity index

Table 2 CMR and MRS findings

\begin{tabular}{|c|c|c|c|c|}
\hline & Controls $\mathrm{N}=12$ & $\mathrm{RA} \mathrm{N}=16$ & SLE N=13 & $P$ value \\
\hline LVEDV indexed to BSA, $\mathrm{ml} / \mathrm{m} 2$ & $78 \pm 15$ & $79 \pm 23$ & $74 \pm 13$ & 0.54 \\
\hline LVESV indexed to BSA, $\mathrm{ml} / \mathrm{m} 2$ & $23 \pm 5$ & $23 \pm 11$ & $21 \pm 6$ & 0.75 \\
\hline LVEF, \% & $72 \pm 4$ & $72 \pm 7$ & $72 \pm 5$ & 0.96 \\
\hline LV Mass indexed to BSA, $\mathrm{g} / \mathrm{m} 2$ & $53 \pm 14$ & $55 \pm 11$ & $47 \pm 7$ & 0.09 \\
\hline LA size, $\mathrm{mm}$ & $27 \pm 5$ & $33 \pm 6$ & $29 \pm 4$ & 0.02 \\
\hline Mid SA circumferential strain & $-18.7 \pm 1.1$ & $-17.5 \pm 0.9$ & $-16.5 \pm 0.8$ & $<0.001$ \\
\hline Peak diastolic circumferential strain rate $(s-1)$ & $118 \pm 21$ & $88 \pm 18$ & $76 \pm 24$ & $<0.001$ \\
\hline Presence of LGE (\%) & 0 & $8(50)$ & $3(23)$ & - \\
\hline Volume fraction of LGE $>2 S D(\%)$ & 0 & $3.4 \pm 0.4$ & $2.6 \pm 0.3$ & - \\
\hline Global myocardial T2 SI Ratio & $1.5 \pm 0.1$ & $1.7 \pm 0.3$ & $1.9 \pm 0.4$ & 0.03 \\
\hline Volume fraction of oedema by T2 (\%) & 0 & $26(18-39)$ & $20(13-31)$ & - \\
\hline Average myocardial $\mathrm{T} 1, \mathrm{~ms}$ & $961 \pm 12$ & $968 \pm 25$ & $987 \pm 26$ & 0.01 \\
\hline Volume fraction of T1>990ms (\%) & $3(1-5)$ & $34(17-55)$ & $41(23-61)$ & $<0.001$ \\
\hline ECV (\%) & $26.5 \pm 2.7$ & $30.4 \pm 3.1$ & $32.2 \pm 4.4$ & $<0.001$ \\
\hline MPRI & $2.0 \pm 0.3$ & $1.6 \pm 0.2$ & $1.5 \pm 0.5$ & $<0.001$ \\
\hline PCr/ATP & $2.0 \pm 0.3$ & $1.5 \pm 0.3$ & $1.4 \pm 0.3$ & $<0.001$ \\
\hline Cardiac lipid content (\%) & $0.49 \pm 0.37$ & $0.46 \pm 0.33$ & $0.58 \pm 0.27$ & 0.78 \\
\hline Hepatic lipid content (\%) & $1.70 \pm 0.72$ & $1.46 \pm 0.58$ & $2.17 \pm 1.02$ & 0.64 \\
\hline
\end{tabular}

Continuous data are mean \pm SD unless otherwise indicated. BSA, body surface area; ECV, extracellular volume; LA, left atrium; LGE, late gadolinium enhancement; LV, left ventricle/ventricular; LVEDV, left ventricular end-diastolic volume; LVEF, left ventricular ejection fraction; LVESV, left ventricular end-systolic volume; MPRI, myocardial perfusion reserve index; PCr/ATP, phosphocreatine to adenosine triphosphate ratio; RPP, rate pressure product; SA, short axis; SI, signal intensity, SLE, systemic lupus erythematosus 
myocardial fibrosis and oedema. There are no differences in myocardial lipid content between patients and controls.

\section{Funding}

This study was funded by investigator-led grants from Guerbet and GlaxoSmithKline.

\section{Authors' details}

'OCMR, Division of Cardiovascular Medicine, University of Oxford, Oxford, UK. ${ }^{2}$ Division of Cardiology, Department of Medicine, University of Cape Town, Cape Town, South Africa. ${ }^{3}$ Division of Brain Sciences, Imperial College London, London, UK. ${ }^{4}$ Bortnar Institute, Nuffield Department of

Orthopaedics, Rheumatology and Musculoskeletal Sciences, University of Oxford, Oxford, South Africa. ${ }^{5}$ Department of Cardiology, University of New South Wales, Sydney, NSW, Australia.

Published: 3 February 2015

doi:10.1186/1532-429X-17-S1-099

Cite this article as: Ntusi et al:: Impaired energetics and normal myocardial lipids in rheumatoid arthritis and systemic lupus erythematosus: a phosphorous and proton magnetic resonance spectroscopy and cardiovascular magnetic resonance study. Journal of Cardiovascular Magnetic Resonance 2015 17(Suppl 1):099.

Submit your next manuscript to BioMed Central and take full advantage of:

- Convenient online submission

- Thorough peer review

- No space constraints or color figure charges

- Immediate publication on acceptance

- Inclusion in PubMed, CAS, Scopus and Google Scholar

- Research which is freely available for redistribution

Submit your manuscript at www.biomedcentral.com/submit
C Biomed Central 